\title{
PI3K/Akt/YAP signaling promotes migration and invasion of DLD-1 colorectal cancer cells
}

\author{
TOMOYA TAKEDA* ${ }^{*}$ YUUTA YAMAMOTO* ${ }^{*}$ MASANOBU TSUBAKI, TAKUYA MATSUDA, \\ AKIHIRO KIMURA, NATSUMI SHIMO and SHOZO NISHIDA
}

Division of Pharmacotherapy, Kindai University School of Pharmacy, Higashi-Osaka, Osaka 577-8502, Japan

Received October 26, 2021; Accepted January 25, 2022

DOI: $10.3892 / \mathrm{ol} .2022 .13226$

\begin{abstract}
Colorectal cancer (CRC) is one of the most prevalent malignant diseases and metastasis is the leading cause of poor prognosis in patients with CRC. Further knowledge of the molecular mechanism underlying metastasis in CRC and the identification of new therapeutic targets are needed. Yes-associated protein (YAP) is a transcriptional regulator that is important in tumorigenesis and tumor cell proliferation. The present study investigated whether YAP was crucial for CRC migration and invasion. The protein expression levels were detected via western blotting, and migration and invasion were analyzed by Transwell migration and invasion assays. Subsequently, YAP expression was silenced using small interfering RNA. The mRNA expression levels were detected via reverse transcription-quantitative PCR and cell viability was assessed via Trypan blue exclusion assay. The results revealed that YAP protein levels were associated with migration and invasion of CRC cells. Notably, YAP small interfering RNA inhibited the migration and invasion of DLD-1 cells. In addition, the phosphoinositide 3-kinase (PI3K)/Akt signaling pathway inhibitor LY294002 suppressed the migration and invasion of DLD-1 cells by decreasing the expression of YAP. Notably, the present study demonstrated that verteporfin mediated the suppression of migration and invasion of DLD-1 cells due to the decreased expression of YAP. Therefore, targeting YAP may be valuable for developing therapeutic strategies
\end{abstract}

Correspondence to: Professor Shozo Nishida, Division of Pharmacotherapy, Kindai University School of Pharmacy, 3-4-1 Kowakae, Higashi-Osaka, Osaka 577-8502, Japan

E-mail: nishida@phar.kindai.ac.jp

*Contributed equally

Abbreviations: CRC, colorectal cancer; YAP, yes-associated protein; PI3K, phosphoinositide 3-kinase; ERK, extracellular signal-regulated kinase; siRNA, small interfering RNA; MEK, mitogen-activated protein kinase kinase; TEAD, transcriptional enhancer associate domain

Key words: CRC, YAP, migration, invasion, verteporfin, PI3K/Akt signaling pathway against CRC, and verteporfin may be an effective therapy to suppress the migration and invasion of CRC.

\section{Introduction}

Colorectal cancer (CRC) is the third most common type of cancer and ranks as the second most frequent cause of cancer-related deaths. The incidence in young individuals continues to increase (1). Tumor metastasis is the leading cause of death in patients with cancer. At least half of all patients with CRC experience systemic metastases. The most frequent metastatic sites are the lungs and liver $(2,3)$. Once metastasis occurs, the clinical outcomes of conventional therapies, including surgery, radiotherapy, chemotherapy, and targeted drug therapy, remain unsatisfactory $(4,5)$. Defining the molecular mechanisms underlying the progression and metastasis of CRC will help to identify novel biomarkers and provide efficient therapeutic strategies to improve CRC treatment.

Yes-associated protein (YAP) is the main effector of Hippo signaling and is the key mechanism in the regulation of cellular proliferation, differentiation, fate determination, and regeneration $(6,7)$. In mammalian systems, YAP translocates from the cytoplasm to the nucleus, where it induces the transcriptional activity of genes associated with cell proliferation, apoptosis, migration, and invasion by interacting with DNA-binding transcription factors $(8,9)$. Accumulating evidence suggests that YAP contributes to the progression in human cancers, including breast cancer, melanoma, and lung cancer (10-12). Aberrant YAP expression or activation is also associated with poor prognosis (13-15). In addition, YAP is frequently overexpressed in CRC tissues and has been correlated with pathological grading, lymph node metastasis, and survival in CRC $(16,17)$. However, it is unclear whether YAP serves as a useful therapeutic target to inhibit CRC metastasis.

In the present study, we tested whether YAP could be the key mechanism involved in CRC migration and invasion. We investigated whether YAP protein levels are correlated with the metastatic phenotype of CRC cells and serve as a useful therapeutic target. Importantly, we found that YAP plays a critical role in the migration and invasion of DLD-1 cells. Furthermore, we also tested the potential of verteporfin, a small molecule that inhibits YAP activation, as a therapeutic agent to inhibit the migration and invasion of DLD-1 cells. The 
collective findings indicate potential therapeutic targets that can help to suppress the migration and invasion of CRC cells.

\section{Materials and methods}

Cell culture. Caco-2, LoVo and Colo-205 cell lines were purchased from the Riken Cell Bank (Ibaraki, Japan). The DLD-1 cell line was obtained from the Health Science Research Resources Bank (Osaka, Japan). These cell lines were grown in RPMI-1640 (Sigma-Aldrich; Merck KGaA) supplemented with 10\% FBS (Gibco; Thermo Fisher Scientific, Inc.).

Chemicals and reagents. Verteporfin was purchased from ChemScene. Trametinib was obtained from LC Laboratories. LY294002 was purchased from Promega. The reagents were dissolved in dimethyl sulfoxide (DMSO). Antibodies against lamin A/C were purchased from Santa Cruz Biotechnology. Antibodies against $\beta$-actin were obtained from Sigma-Aldrich; Merck KGaA. Antibodies against YAP, phosphorylated (p)-Akt, Akt, p-extracellular signal-regulated kinase (ERK), and ERK were obtained from Cell Signaling Technology. Small interfering RNA (siRNA) targeting YAP (HSS115942); (5'-GCA ACT CCA ACC AGCAGCA ACAGAT-3') was purchased from Thermo Fisher Scientific, Inc.

Silencing of YAP. DLD-1 cells were transfected with YAP siRNA $(10,20$ and $50 \mathrm{nM})$ or Stealth ${ }^{\mathrm{TM}}$ RNAi Negative Control (NC) (Invitrogen; Thermo Fisher Scientific, Inc.) using Lipofectamine 3000 (Invitrogen; Thermo Fisher Scientific, Inc.). After transfection, the cells were treated according to the experimental requirements.

Total RNA extraction and reverse transcription-quantitative $P C R$. Total RNA was extracted using RNAiso Plus reagent (Takara Bio) according to the manufacturer's instructions. The RNA was reverse-transcribed to cDNA using the PrimeScript ${ }^{\mathrm{TM}}$ RT reagent kit (Takara Bio), according to the manufacturer's protocol. Quantitative PCR was performed with the Thermal Cycler Dice Real Time system (Takara Bio) using SYBR Premix Ex Taq (Takara Bio). The PCR conditions were an initial denaturation at $95^{\circ} \mathrm{C}$ for $5 \mathrm{~min}$, followed by 40 cycles of denaturation at $94^{\circ} \mathrm{C}$ for $30 \mathrm{sec}$, annealing at $50^{\circ} \mathrm{C}$ for $30 \mathrm{sec}$, and extension at $72^{\circ} \mathrm{C}$ for $30 \mathrm{sec}$. The following primers were used: YAP forward, 5'-CCTCGTTTTGCCATGAACCAG-3' and reverse, 5'-GTTCTTGCTGTTTCAGCCGCAG-3'; glyceraldehyde 2-phosphate dehydrogenase (GAPDH) forward, 5'-AAGGTCGGAGTCAACGGATT-3' and reverse, 5'-CTC CTGGAAGATGGTGATGG-3'. The expression levels were normalized to the GAPDH internal control and fold changes in expression levels were calculated using the $2^{-\Delta \Delta \mathrm{Cq}}$ method.

Western blot analysis. Western blot analysis was performed as previously described (18). The cells were lysed using lysis buffer, and the protein concentration was determined using the BCA protein assay kit (Thermo Fisher Scientific, Inc.). Proteins were separated using sodium dodecyl sulfate-polyacrylamide gel electrophoresis and subsequently transferred to polyvinylidene fluoride membranes (EMD Millipore). The membranes were treated with primary antibodies at $4^{\circ} \mathrm{C}$ overnight, followed by incubation with the appropriate secondary antibody. The proteins were detected using the Luminata Forte Western HRP Substrate (EMD Millipore).

Transwell migration and invasion assay. Transwell migration and invasion assays were performed as described previously (19). DLD-1 cells were treated for $24 \mathrm{~h}$ with YAP siRNA (10 and $20 \mathrm{nM})$, verteporfin $(10,50$ and $100 \mathrm{nM})$, and LY294002 $(1$ and $5 \mu \mathrm{M})$. Cells $\left(2 \times 10^{4}\right)$ were collected and seeded in the upper chamber without Matrigel coating for the migration assay and in the upper chamber precoated with Matrigel for the invasion assay. After $24 \mathrm{~h}$, cells that had traversed the membrane were counted using a light microscope (Olympus).

Trypan blue exclusion assay. DLD-1 cells $\left(2 \times 10^{4}\right)$ were treated with YAP siRNA $(10,20$ and $50 \mathrm{nM})$, verteporfin (10, 50 and $100 \mathrm{nM}), \mathrm{LY} 294002(1,5$ and $10 \mu \mathrm{M})$, and trametinib (1 and $10 \mu \mathrm{M}$ ). After $72 \mathrm{~h}$ of incubation, the number of stained cells was counted. There are no images of trypan blue exclusion assay in this study.

Statistical analysis. All experiments were repeated three times. The results are expressed as the mean \pm standard deviation (SD). Statistical analysis involved analysis of variance (ANOVA) with Dunnett's test. Statistical significance was set at $\mathrm{P}<0.05$.

\section{Results}

Levels of YAP expression are correlated with migration and invasion of CRC cells. We examined whether high levels of YAP correlated with the metastatic phenotype of cells from four human CRC cell lines (Caco-2, LoVo, Colo-205, and DLD-1). We first examined the expression of YAP in the CRC cells using western blot analysis. Only DLD-1 cells produced a high level of YAP protein (Fig. 1A). We next investigated the migration and invasion of the four CRC cell lines using the Transwell migration and invasion assay. Migration and invasion were more pronounced for DLD-1 cells compared to those of Caco-2, LoVo, and Colo-205 cells (Figs. 1B and C, and S1). These results support the view that the levels of YAP protein are correlated with high migration and invasion in CRC cell lines. The DLD-1 cells that abundantly expressed YAP and displayed a highly metastatic phenotype were used for subsequent experiments.

YAP promotes migration and invasion of DLD-1 cells. To determine the contribution of YAP to migration and invasion, these attributes of DLD-1 cells were examined when YAP was silenced using siRNA. DLD-1 cells were transfected with siRNA specific for YAP in DLD-1, and the mRNA and protein expression levels of YAP were determined after 2 days. The levels of YAP mRNA and protein were suppressed in DLD-1 cells treated with YAP siRNA in a concentration-dependent manner (Fig. 2A and B). Next, the effect of YAP siRNA on the viability of DLD-1 cells was assessed using the Trypan blue exclusion assay. DLD-1 cells treated with 10 and $20 \mathrm{nM}$ YAP siRNA showed no inhibition of cell viability (Fig. 2C). However, $50 \mathrm{nM}$ YAP siRNA reduced the viability of DLD-1 cells compared to that of untreated cells. These conditions 
A
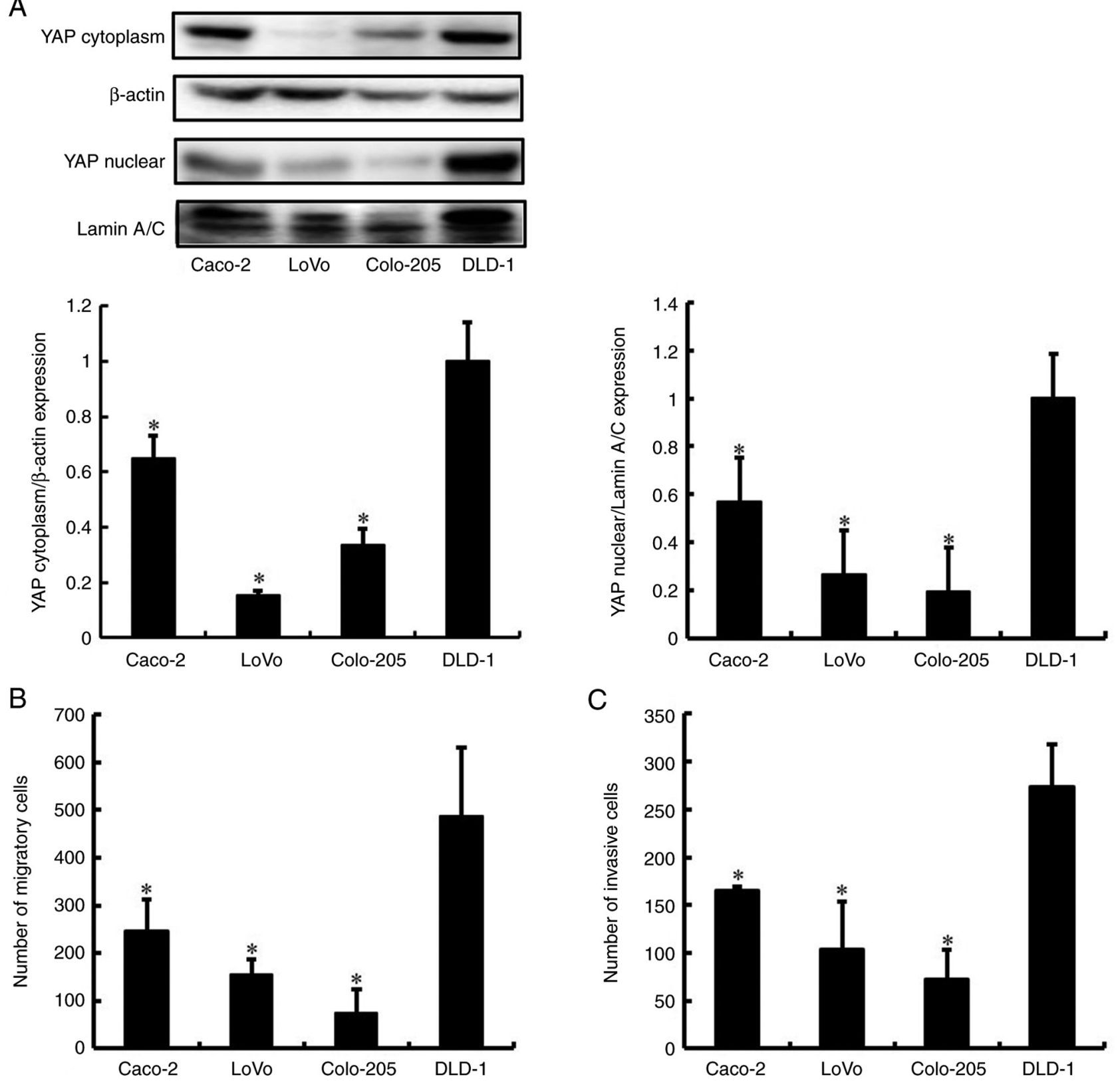

Figure 1. The levels of YAP expression are correlated with pronounced migration and invasion of CRC cell lines. (A) The expression of YAP in CRC cell lines (Caco-2, LoVo, Colo-205, and DLD-1) was detected by western blot. $\beta$-actin and Lamin A/C was analyzed as an internal control. Bands were normalized to that of $\beta$-actin and Lamin A/C. (B) Migration was analyzed using Transwell culture inserts. (C) Invasion was analyzed using Transwell culture inserts coated with Matrigel. Data are represented as mean \pm SD and have been repeated three times with similar results. ${ }^{*}<0.05$ compared to DLD-1. YAP, yes-associated protein; CRC, colorectal cancer; SD, standard deviation.

were used for subsequent experiments to assess the effects of YAP siRNA on the migration and invasion of DLD-1 cells. YAP siRNA inhibited the migration and invasion of DLD-1 cells but did not affect viability (Fig. 2D and E, and S2). Although the phenotypes observed after depletion of YAP mRNA and proteins with YAP siRNAs are usually attributed to the impaired function of these proteins, it is possible that they are due to off-target effects of the siRNAs. These results suggest that YAP is important for the migration and invasion of DLD-1 cells.

PI3K/Akt pathway regulates YAP activation and promotes migration and invasion of DLD-1 cells. YAP is regulated by signaling pathways, including mitogen-activated protein kinase (MAPK)/extracellular signal-regulated kinase (ERK) and phosphoinositide 3-kinase (PI3K)/Akt pathways $(20,21)$. In addition, we previously reported that the YAP-high expressing DLD-1 cell line harbors a K-Ras mutation and phosphatidylinositol-4,5-bisphosphate 3-kinase catalytic subunit alpha (PIK3CA) mutations (22). To determine whether the PI3K/Akt and/or MEK/ERK pathways regulate YAP activation and therefore promote the migration and invasion of DLD-1 cells, we examined the expression of YAP in cells treated with the PI3K/Akt signaling pathway inhibitor LY294002 and the MEK/ERK signaling pathway inhibitor trametinib. LY294002 suppressed YAP activation 
A
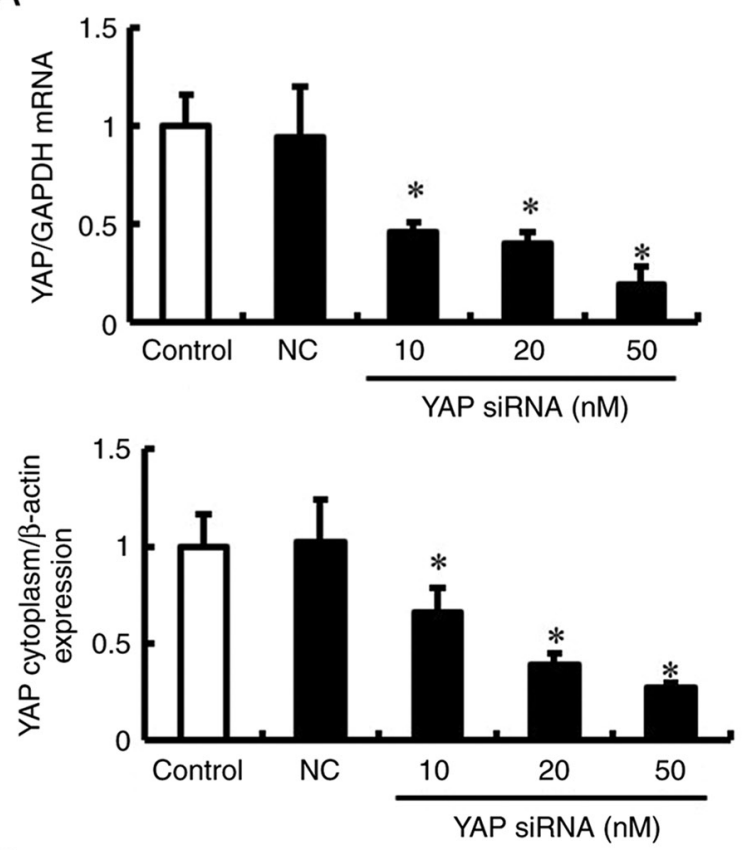

C

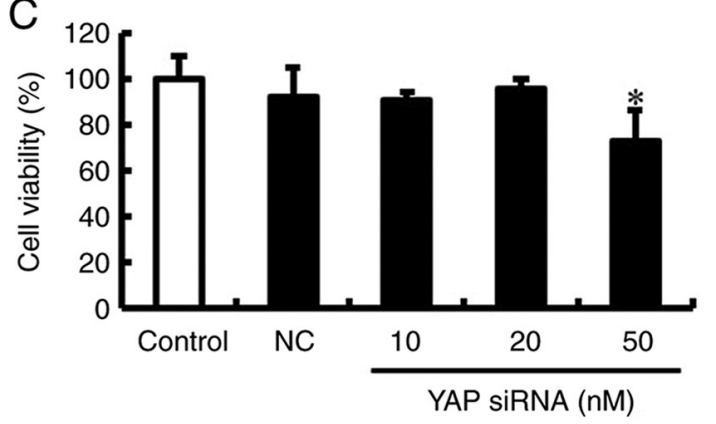

B

YAP cytoplasm
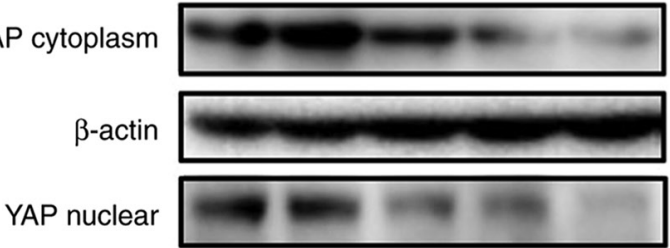

Lamin $\mathrm{A} / \mathrm{C}$
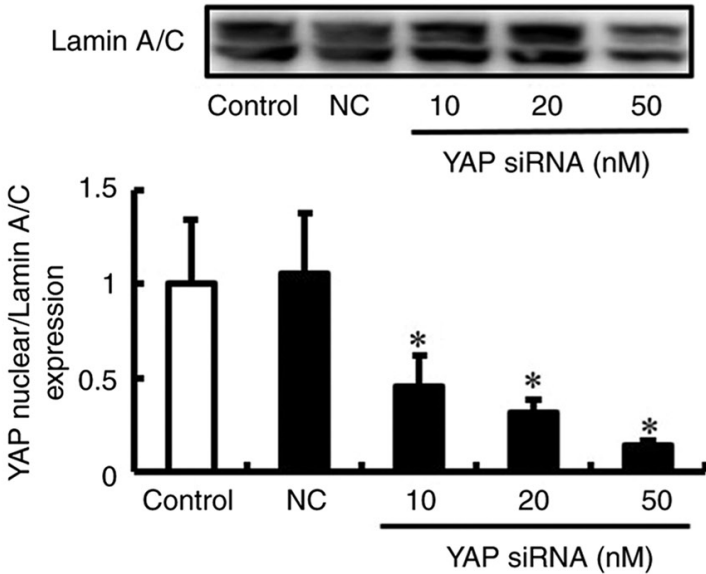

$\mathrm{D}$

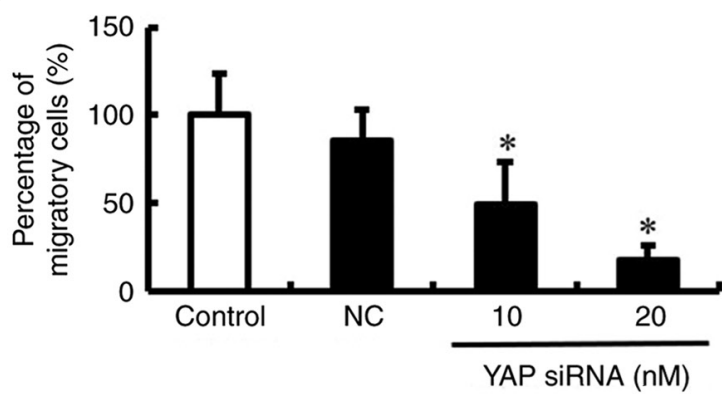

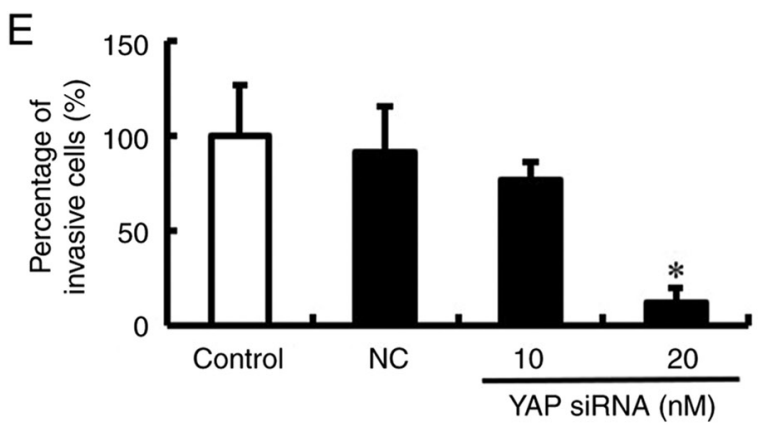

Figure 2. YAP promotes migration and invasion of DLD-1 cells. (A-C) DLD-1 cells were treated with YAP siRNA (10, 20 and 50 nM), Stealth ${ }^{\mathrm{TM}}$ RNAi Negative Control (NC), or were not treated with siRNA (control). (A) The mRNA expression of YAP mRNA was measured by reverse transcription-quantitative PCR. The expression levels were normalized to the internal level of GAPDH. (B) The expression of YAP was detected by western blot. $\beta$-actin and Lamin A/C was analyzed as an internal control. Bands were normalized to that of $\beta$-actin and Lamin A/C. (C) Cell viability was determined by trypan blue. (D and E) DLD-1 cells were treated with YAP siRNA (10 and $20 \mathrm{nM}$ ), Stealth ${ }^{\mathrm{TM}}$ RNAi Negative Control (NC) or were not treated with siRNA (control). (D) Migration was analyzed using Transwell culture inserts. (E) Invasion was analyzed using Transwell culture inserts coated with Matrigel. The percentages of migratory and invasive cells are shown relative to the control group. Data are represented as mean $\pm \mathrm{SD}$ and have been repeated three times with similar results. "P<0.05 compared with control. GAPDH, glyceraldehyde-3-phosphate dehydrogenase. YAP, yes-associated protein; siRNA, small interfering RNA; GAPDH, glyceraldehyde 2-phosphate dehydrogenase; SD, standard deviation.

by inhibiting Akt phosphorylation (Fig. 3A). However, no change was observed in YAP activation upon inhibition of ERK phosphorylation by trametinib (Fig. 3B). Next, we tested the effect of LY294002 on the viability of DLD-1 cells. Viability was not reduced in DLD-1 cells treated with 1 and $5 \mu \mathrm{M}$ LY294002 (Fig. 4A). However, $10 \mu \mathrm{M}$ LY294002 reduced the viability of DLD-1 cells compared to that of untreated cells. These conditions were used in subsequent experiments to assess the effects of LY294002 on the migration and invasion of DLD-1 cells. LY294002 inhibited the migration and invasion of DLD-1 cells but did not affect viability (Figs. 4B and C, and S3). These results showed that the PI3K/Akt pathway regulates YAP activation and promotes the migration and invasion of DLD-1 cells. 

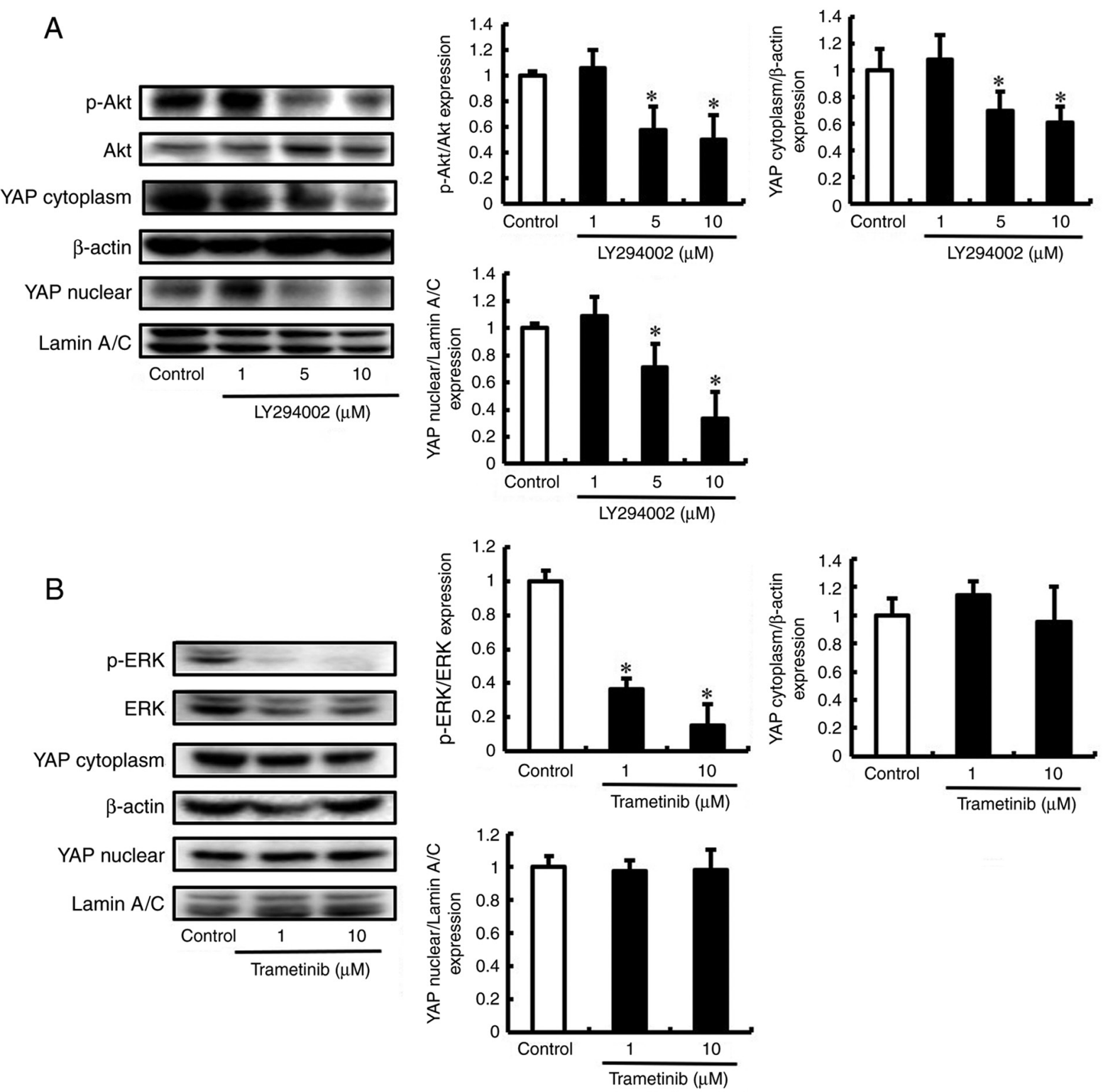

Figure 3. LY294002 suppresses YAP activation by inhibiting Akt phosphorylation. (A) DLD-1 cells were untreated (control) or treated with LY294002 (1, 5 and $10 \mu \mathrm{M}$ ). The expression of YAP, p-Akt, and Akt was detected by western blot. $\beta$-actin and Lamin A/C were analyzed as an internal control. Bands were normalized to that of Akt, $\beta$-actin and Lamin A/C. (B) DLD-1 cells were untreated (control) or treated with trametinib (1 and $10 \mu \mathrm{M})$. The expression of YAP, p-ERK, and ERK was detected by western blot. $\beta$-actin and Lamin $\mathrm{A} / \mathrm{C}$ were analyzed as an internal control. Bands were normalized to that of ERK, $\beta$-actin and Lamin A/C. Data are mean \pm SD and have been repeated three times with similar results. "P $<0.05$ compared with control. YAP, yes-associated protein; ERK, extracellular signal-regulated kinase; SD, standard deviation.

Verteporfin suppresses migration and invasion of DLD-1 cells by decreasing YAP production. Verteporfin is primarily used as a photosensitizer for the treatment of choroidal neovascularization in age-related macular degeneration in ophthalmology (23). Previous studies have reported that verteporfin inhibits YAP activation by preventing its binding to the transcriptional enhancer associate domain (TEAD) (24). Thus, we investigated the potential of repurposing verteporfin as a new treatment for metastatic CRC. The expression of YAP in cells treated with verteporfin was assessed by western blot analysis. Verteporfin decreased the production of YAP in DLD-1 cells in a concentration-dependent manner (Fig. 5A). Next, the effect of verteporfin on the viability of DLD-1 cells was assessed using the Trypan blue exclusion assay. Viability was not significantly decreased in DLD-1 cells treated with 10 ,
50 and $100 \mathrm{nM}$ verteporfin (Fig. 5B). The effects of verteporfin on the migration and invasion of DLD-1 cells were also examined. Consistent with YAP knockdown, verteporfin treated DLD-1 cells exhibited significantly decreased migration and invasion (Figs. 5C and D, and S4). These results indicate that verteporfin inhibits the expression of YAP and suppresses the migration and invasion of DLD-1 cells.

\section{Discussion}

CRC is one of the most prevalent malignant tumors. Its poor prognosis is mainly ascribed to the pronounced malignant invasion and metastasis of the cancer cells. Clarifying the mechanism of metastasis could facilitate the design of novel and more effective therapeutic strategies for metastasis in 

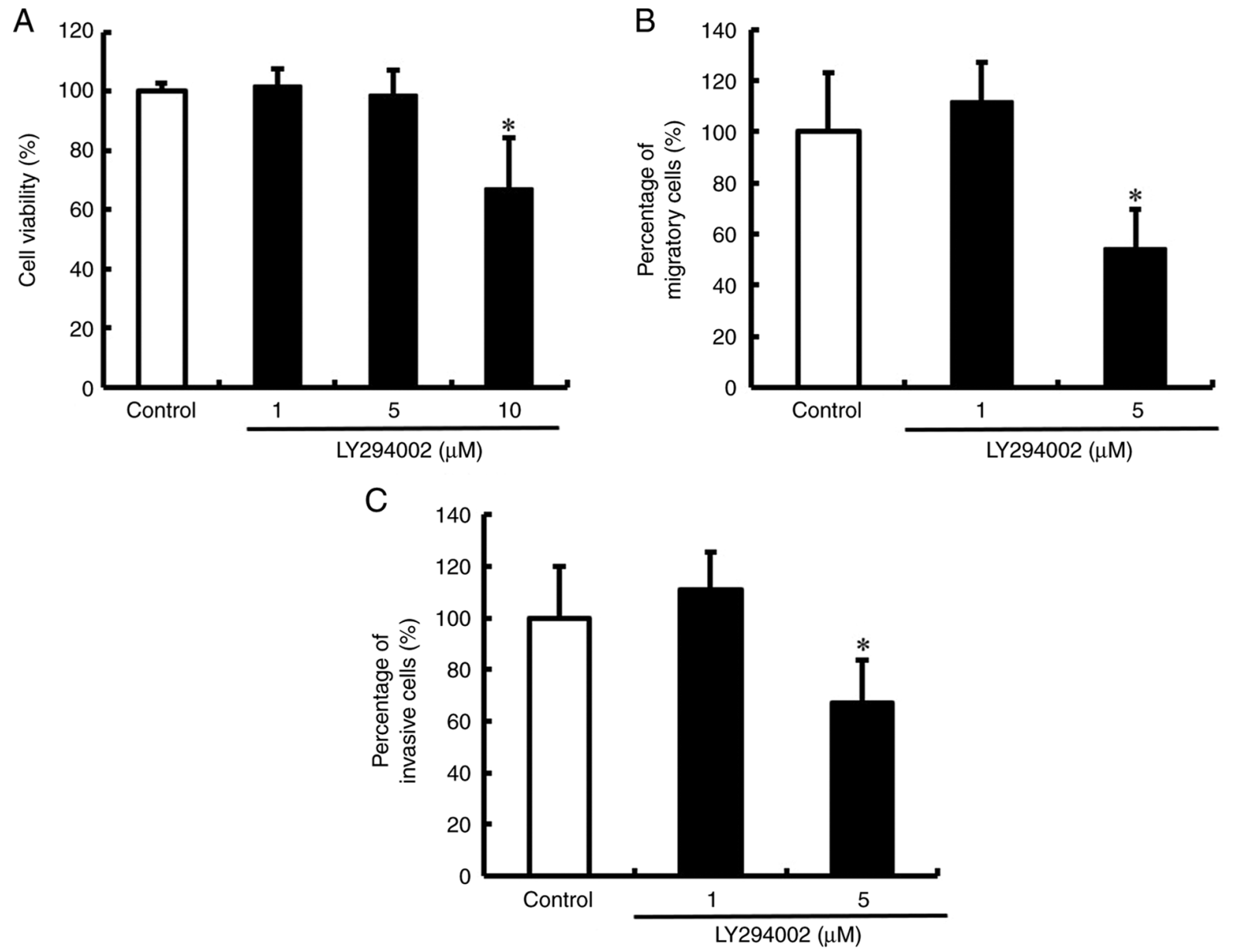

Figure 4. LY294002 decreases migration and invasion of DLD-1 cells. (A) DLD-1 cells were untreated (control) or treated with LY294002 (1, 5 and $10 \mu$ M). Cell viability was determined by trypan blue. (B and C) DLD-1 cells were untreated (control) or treated with LY294002 (1 and $5 \mu \mathrm{M}$ ). (B) Migration was analyzed using Transwell culture inserts. (C) Invasion was analyzed using Transwell culture inserts coated with Matrigel. The percentages of migratory and invasive cells are shown relative to the control group. Data are mean $\pm \mathrm{SD}$ and have been repeated three times with similar results. "P<0.05 compared with control. YAP, yes-associated protein; SD, standard deviation.

CRC patients. Numerous studies have confirmed that dysregulation of signaling pathways is involved in the migration, invasion, and metastasis of human cancers (25-28). YAP is involved in the regulation of tissue growth, homeostasis, and tumor development (29). Altered expression of YAP has been reported in various cancers that include breast, ovarian, and liver cancers. The expression levels of YAP are associated with disease-free survival (DFS) and overall survival (OS) of patients with tumors $(30,31)$. In this study, we investigated whether YAP protein levels are correlated with the metastatic phenotype of CRC cells and thus have potential as a useful therapeutic target. The level of YAP protein was correlated with pronounced migration and invasion of CRC cells. Inhibition of YAP expression decreased the migration and invasion of DLD-1 cells. Consistent with these findings, inhibition of YAP reportedly suppressed the migration and invasion of pancreatic cancer cells (32). These results suggest that YAP plays an important role in the migration and invasion of DLD-1 cells.

Although an increasing number of negative regulators of YAP have been identified, there are few known positive regulators of YAP $(21,33)$. Recent studies have reported that the MEK/ERK and PI3K/Akt pathways positively regulate YAP activation $(34,35)$. In this study, we used the YAP high expression cell line DLD-1, which harbors KRAS and PIK3CA mutations. KRAS mutations are frequent in CRC and have the potential to activate proliferation, survival, migration, and invasion through MEK/ERK signaling pathways (36). PIK3CA mutation also leads to the activation of the PI3K/Akt signaling pathway, promoting cancer growth in CRC (37). Presently, the PI3K/Akt signaling pathway inhibitor LY294002 suppressed YAP activation by inhibiting Akt phosphorylation. Furthermore, LY294002 inhibited the migration and invasion of DLD-1 cells. These results indicate that the PI3K/Akt pathway regulates YAP activation and promotes the migration and invasion of DLD-1 cells.

Drug repositioning refers to the discovery of new indications for drugs that are clinically approved for other indications. For approved drugs that have been clinically used for a long time, the dosage, safety, dosage, safety, toxicity, tolerability, and pharmacokinetic features are clear. In addition, the success rate of drug repurposing approaches accounts for approximately 30\% of new Food and Drug Administration-approved (FDA) drugs and vaccines in recent years (38). Therefore, repurposed candidate drugs reduce the time and costs associated with drug development. Verteporfin 
A
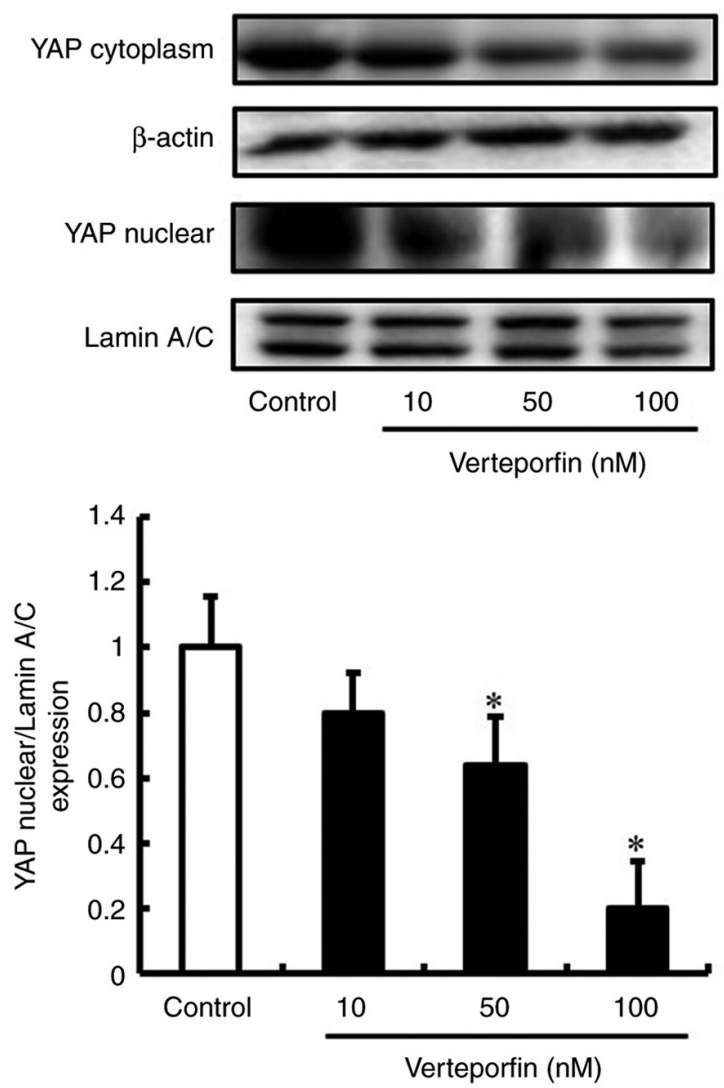

C

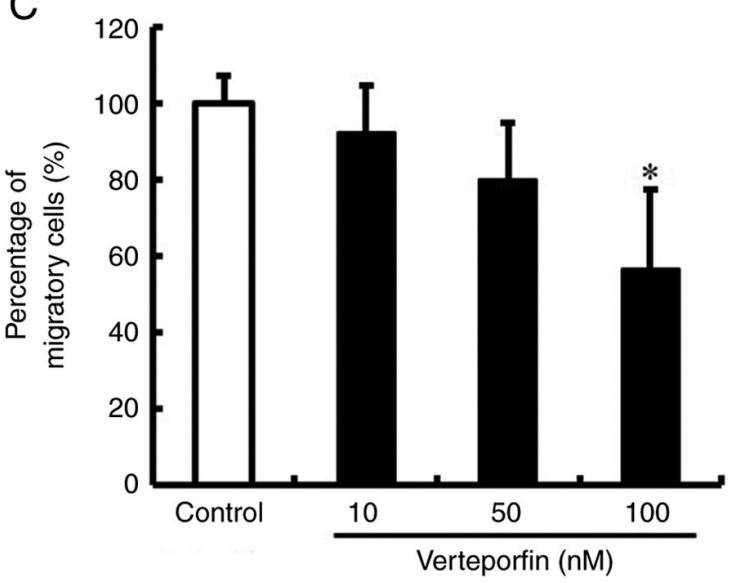

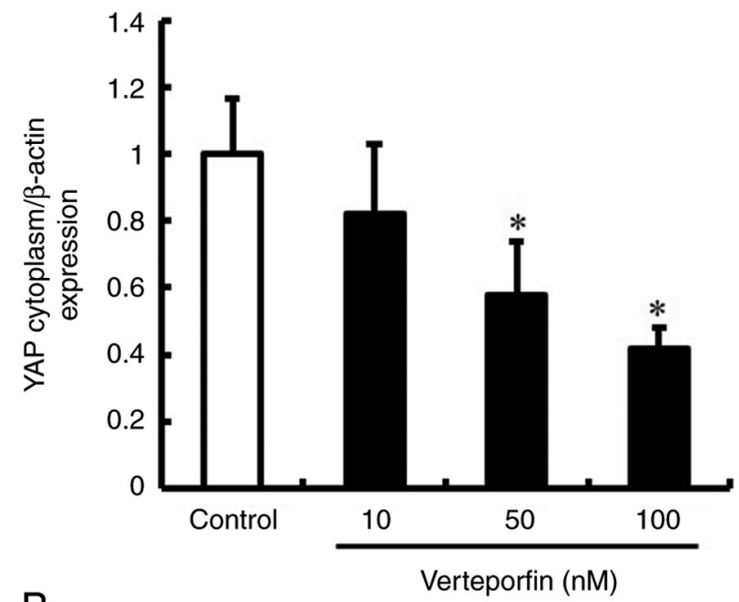

B
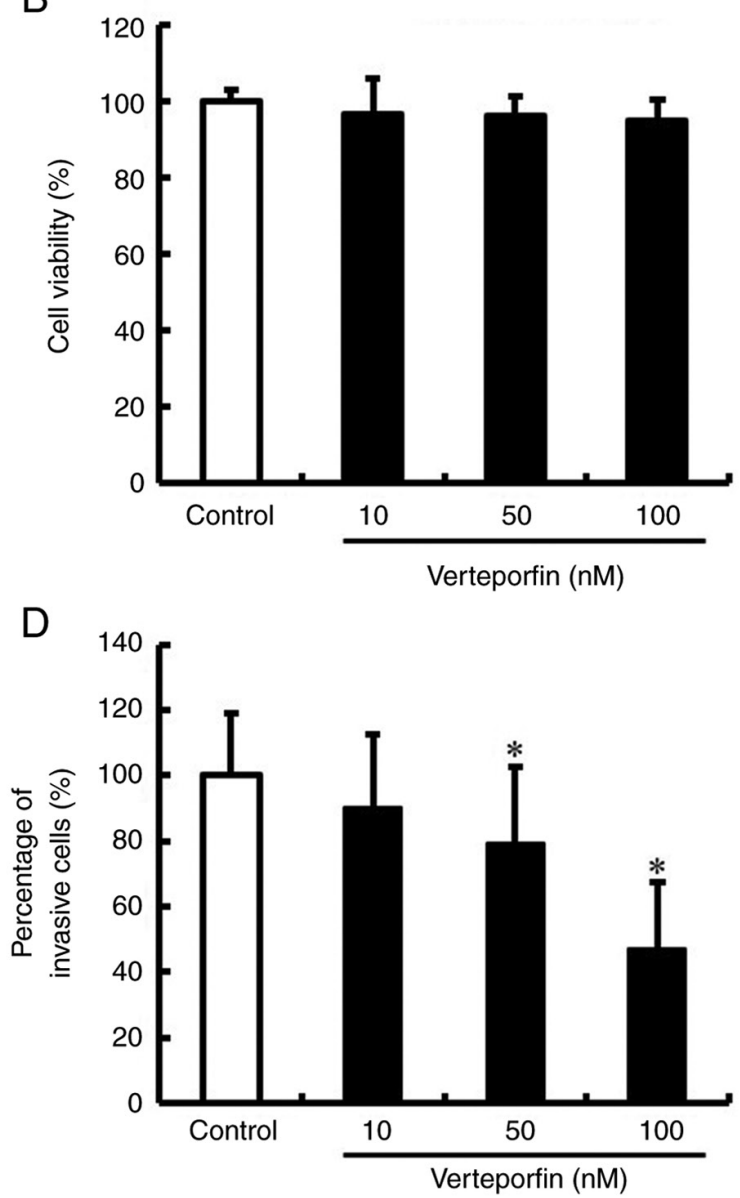

Figure 5. Verteporfin suppresses the migration and invasion of DLD-1 cells by decreasing the expression of YAP. DLD-1 cells were untreated (control) or treated with verteporfin (10,50 and $100 \mathrm{nM})$. (A) The expression of YAP was detected by western blot. $\beta$-actin and Lamin A/C was used as an internal control. Quantification of the amount of YAP was normalized to the amount of $\beta$-actin and Lamin A/C. (B) Cell viability was determined by trypan blue. (C) Migration was analyzed using Transwell culture inserts. (D) Invasion was analyzed using Transwell culture inserts coated with Matrigel. The percentages of migratory and invasive cells are shown relative to the control group. Data are represented as mean $\pm \mathrm{SD}$ and have been repeated three times with similar results. ${ }^{*} \mathrm{P}<0.05$ compared with control. YAP, yes-associated protein; SD, standard deviation.

is a second-generation photosensitizer approved by the FDA for photodynamic therapy in macular degeneration as a photosensitizer (39). The present evidence demonstrates that verteporfin suppresses the migration and invasion of DLD-1 cells by decreasing YAP expression. Verteporfin was recently identified as a disruptor of YAP-TEAD-mediated transcription, which inhibits the YAP-TEAD complex (40). Moreover, verteporfin was reported to decrease the expression of YAP protein by increasing the levels of 14-3-3 $\sigma$, a YAP chaperone protein (24). These findings support the use of verteporfin as an effective therapy to suppress CRC migration and invasion.

This study has a few limitations. Migration and invasion of cells are important factors in cancer cell metastasis. In this study, we showed that inhibition of YAP suppresses the migration and invasion of DLD-1 cells. However, we have 
only circumstantial evidence to support the relevance of our findings in vivo. Further studies are warranted to scientifically establish the efficacy of YAP inhibition in DLD-1 cells in vivo. Moreover, the present experiments involved the DLD-1 human CRC cell line. These results were not confirmed in other human CRC cell lines. The contribution of YAP to migration, invasion, and metastasis should be more widely studied in other human CRC cell lines.

In summary, the levels of YAP protein were correlated with high migration and invasion of CRC cells. YAP siRNA inhibited the migration and invasion of DLD-1 cells, but did not influence viability. Furthermore, the Akt inhibitor LY294002 suppressed YAP activation by inhibiting Akt phosphorylation and decreasing the migration and invasion of DLD-1 cells. Importantly, verteporfin suppressed the migration and invasion of DLD-1 cells by decreasing the expression of YAP. The collective findings indicate that targeting YAP might be valuable for developing therapeutics against CRC. Verteporfin may be an effective therapy to suppress the migration and invasion of CRC.

\section{Acknowledgements}

Not applicable.

\section{Funding}

This study was supported in part by a Grant-in-Aid for Young Scientists from the Japan Society for the Promotion of Science (JSPS) (grant no. 20K16343).

\section{Availability of data and materials}

The datasets used and/or analyzed during the current study are available from the corresponding author on reasonable request.

\section{Authors' contributions}

SN, TT and, YY designed the study. TT wrote the manuscript, and performed western blot analysis and silencing of YAP. YY edited the manuscript, performed western blot analysis, trypan blue exclusion assay and silencing of YAP. MT performed the trypan blue exclusion assay and statistical analysis. TM, AK and NS performed the Transwell migration and invasion assays, western blot analysis and statistical analysis. SN, TT and YY confirm the authenticity of all raw data. All authors read, revised, and approved the final manuscript.

\section{Ethics approval and consent to participate}

Not applicable.

\section{Patient consent for publication}

Not applicable.

\section{Competing interests}

The authors declare that they have no competing interests.

\section{References}

1. Bray F, Ferlay J, Soerjomataram I, Siegel RL, Torre LA and Jemal A: Global cancer statistics 2018: GLOBOCAN estimates of incidence and mortality worldwide for 36 cancers in 185 countries. CA Cancer J Clin 68: 394-424, 2018.

2. Tang M, Wang H, Cao Y,Zeng Z, Shan X and Wang L: Nomogram for predicting occurrence and prognosis of liver metastasis in colorectal cancer: A population-based study. Int J Colorectal Dis 36: 271-282, 2021.

3. Huang Y, Zhao M, Yin J, Lu T, Yang X, Yuan G, Li M, Liu Y, Zhan C and Wang Q: Pulmonary metastasis in newly diagnosed colon-rectal cancer: A population-based nomogram study. Int J Colorectal Dis 34: 867-878, 2019.

4. Cao LL, Pei XF, Qiao X, Yu J, Ye H, Xi CL, Wang PY and Gong ZL: SERPINA3 silencing inhibits the migration, invasion, and liver metastasis of colon cancer cells. Dig Dis Sci 63: 2309-2319, 2018.

5. Ding YL, Wang QS, Zhao WM and Xiang L: Expression of smoothened protein in colon cancer and its prognostic value for postoperative liver metastasis. Asian Pac J Cancer Prev 13: 4001-4005, 2012.

6. Dobrokhotov O, Samsonov M, Sokabe M and Hirata H: Mechanoregulation and pathology of YAP/TAZ via Hippo and non-Hippo mechanisms. Clin Transl Med 7: 23, 2018.

7. Totaro A, Panciera T and Piccolo S: YAP/TAZ upstream signals and downstream responses. Nat Cell Biol 20: 888-899, 2018.

8. Kim MK, Jang JW and Bae SC: DNA binding partners of YAP/TAZ. BMB Rep 51: 126-133, 2018.

9. Li L, Ugalde AP, Scheele CLGJ, Dieter SM, Nagel R, Ma J, Pataskar A, Korkmaz G, Elkon R, Chien MP, et al: A comprehensive enhancer screen identifies TRAM2 as a key and novel mediator of YAP oncogenesis. Genome Biol 22: 54, 2021.

10. Tsubaki M, Genno S, Takeda T, Matsuda T, Kimura N, Yamashita Y, Morii Y, Shimomura K and Nishida S: Rhosin suppressed tumor cell metastasis through inhibition of Rho/YAP pathway and expression of RHAMM and CXCR4 in melanoma and breast cancer cells. Biomedicines 9: 35, 2021.

11. Feng X, Arang N, Rigiracciolo DC, Lee JS, Yeerna H, Wang Z, Lubrano S, Kishore A, Pachter JA, König GM, et al: A platform of synthetic lethal gene interaction networks reveals that the GNAQ uveal melanoma oncogene controls the Hippo pathway through FAK. Cancer Cell 35: 457-472.e5, 2019.

12. Hsu PC, Tian B, Yang YL, Wang YC, Liu S, Urisman A, Yang CT, Xu Z, Jablons DM and You L: Cucurbitacin E inhibits the Yes-associated protein signaling pathway and suppresses brain metastasis of human non-small cell lung cancer in a murine model. Oncol Rep 42: 697-707, 2019.

13. Warren JSA, Xiao Y and Lamar JM: YAP/TAZ activation as a target for treating metastatic cancer. Cancers (Basel) 10: 115, 2018.

14. Gao J, Han W, He Y, Zhou J, Miao J and Zhang G: Livin promotes tumor progression through YAP activation in ovarian cancer. Am J Cancer Res 10: 3179-3193, 2020.

15. Xu Z, Wang H, Gao L, Zhang $\mathrm{H}$ and Wang X: YAP levels combined with plasma CEA levels are prognostic biomarkers for early-clinical-stage patients of colorectal cancer. Biomed Res Int 2019: 2170830, 2019.

16. Wang L, Shi S, Guo Z, Zhang X, Han S, Yang A, Wen W and Zhu Q: Overexpression of YAP and TAZ is an independent predictor of prognosis in colorectal cancer and related to the proliferation and metastasis of colon cancer cells. PLoS One 8: e65539, 2013.

17. Mouillet-Richard S and Laurent-Puig P: YAP/TAZ signalling in colorectal cancer: Lessons from consensus molecular subtypes. Cancers (Basel) 12: 3160, 2020.

18. Tsubaki M, Ogawa N, Takeda T, Sakamoto K, Shimaoka H, Fujita A, Itoh T, Imano M, Satou T and Nishida S: Dimethyl fumarate induces apoptosis of hematopoietic tumor cells via inhibition of NF- $\kappa \mathrm{B}$ nuclear translocation and down-regulation of Bcl-xL and XIAP. Biomed Pharmacother 68: 999-1005, 2014.

19. Tsubaki M, Komai M,Itoh T,Imano M, Sakamoto K, Shimaoka H, Ogawa N, Mashimo K, Fujiwara D, Takeda T, et al: Inhibition of the tumour necrosis factor-alpha autocrine loop enhances the sensitivity of multiple myeloma cells to anticancer drugs. Eur J Cancer 49: 3708-3717, 2013.

20. You B, Yang YL, Xu Z, Dai Y, Liu S, Mao JH, Tetsu O, Li H, Jablons DM and You L: Inhibition of ERK1/2 down-regulates the Hippo/YAP signaling pathway in human NSCLC cells. Oncotarget 6: 4357-4368, 2015. 
21. Zhao Y, Montminy T, Azad T, Lightbody E, Hao Y, SenGupta S, Asselin E, Nicol C and Yang X: PI3K positively regulates YAP and TAZ in mammary tumorigenesis through multiple signaling pathways. Mol Cancer Res 16: 1046-1058, 2018.

22. Tsubaki M, Takeda T, Noguchi M, Jinushi M, Seki S, Morii Y, Shimomura K, Imano M, Satou T and Nishida S: Overactivation of Akt Contributes to MEK inhibitor primary and acquired resistance in colorectal cancer cells. Cancers (Basel) 11: 1866, 2019.

23. Liu-Chittenden Y, Huang B, Shim JS, Chen Q, Lee SJ, Anders RA, Liu JO and Pan D: Genetic and pharmacological disruption of the TEAD-YAP complex suppresses the oncogenic activity of YAP. Genes Dev 26: 1300-1305, 2012.

24. Wang C, Zhu X, Feng W, Yu Y, Jeong K, Guo W, Lu Y and Mills GB: Verteporfin inhibits YAP function through up-regulating 14-3-3 $\sigma$ sequestering YAP in the cytoplasm. Am J Cancer Res 6: 27-37, eCollection 2016.2015.

25. Leber MF and Efferth T: Molecular principles of cancer invasion and metastasis (review). Int J Oncol 34: 881-895, 2009.

26. Perlikos F, Harrington KJ and Syrigos KN: Key molecular mechanisms in lung cancer invasion and metastasis: A comprehensive review. Crit Rev Oncol Hematol 87: 1-11, 2013.

27. Ming H, Li B, Zhou L, Goel A and Huang C: Long non-coding RNAs and cancer metastasis: Molecular basis and therapeutic implications. Biochim Biophys Acta Rev Cancer 1875: 188519, 2021.

28. Takeda T, Tsubaki M, Asano R, Itoh T, Imano M, Satou T and Nishida S: Dimethyl fumarate suppresses metastasis and growth of melanoma cells by inhibiting the nuclear translocation of NF-кB. J Dermatol Sci 99: 168-176, 2020.

29. Coffey K: Targeting the Hippo pathway in prostate cancer: What's new?. Cancers (Basel) 13: 611, 2021

30. Thompson BJ: YAP/TAZ: Drivers of tumor growth, metastasis, and resistance to therapy. Bioessays 42: e1900162, 2020.

31. Sorrentino G, Ruggeri N, Zannini A, Ingallina E, Bertolio R, Marotta C, Neri C, Cappuzzello E, Forcato M, Rosato A, et al: Glucocorticoid receptor signalling activates YAP in breast cancer. Nat Commun 8: 14073, 2017.
32. Yang S, Zhang L, Purohit V, Shukla SK, Chen X, Yu F, Fu K, Chen Y, Solheim J, Singh PK, et al: Active YAP promotes pancreatic cancer cell motility, invasion and tumorigenesis in a mitotic phosphorylation-dependent manner through LPAR3. Oncotarget 6: 36019-36031, 2015.

33. Yu FX, Zhao B and Guan KL: Hippo pathway in organ size control, tissue homeostasis, and cancer. Cell 163: 811-828, 2015.

34. Li XJ, Leem SH, Park MH and Kim SM: Regulation of YAP through an Akt-dependent process by 3, 3'-diindolylmethane in human colon cancer cells. Int J Oncol 43: 1992-1998, 2013.

35. Qin X, Li J, Sun J, Liu L, Chen D and Liu Y: Low shear stress induces ERK nuclear localization and YAP activation to control the proliferation of breast cancer cells. Biochem Biophys Res Commun 510: 219-223, 2019.

36. Wang $\mathrm{C}$ and Fakih M: Targeting KRAS in colorectal cancer. Curr Oncol Rep 23: 28, 2021

37. Zhu YF, Yu BH, Li DL, Ke HL, Guo XZ and Xiao XY: PI3K expression and PIK3CA mutations are related to colorectal cancer metastases. World J Gastroenterol 18: 3745-3751, 2012.

38. Pillaiyar T, Meenakshisundaram S, Manickam $M$ and Sankaranarayanan M: A medicinal chemistry perspective of drug repositioning: Recent advances and challenges in drug discovery. Eur J Med Chem 195: 112275, 2020.

39. Miller JW, Schmidt-Erfurth U, Sickenberg M, Pournaras CJ, Laqua H, Barbazetto I, Zografos L, Piguet B, Donati G, Lane AM, et al: Photodynamic therapy with verteporfin for choroidal neovascularization caused by age-related macular degeneration: Results of a single treatment in a phase 1 and 2 study. Arch Ophthalmol 117: 1161-1173, 1999.

40. Brodowska K, Al-Moujahed A, Marmalidou A, Meyer Zu Horste M, Cichy J, Miller JW, Gragoudas E and Vavvas DG: The clinically used photosensitizer Verteporfin (VP) inhibits YAP-TEAD and human retinoblastoma cell growth in vitro without light activation. Exp Eye Res 124: 67-73, 2014. 